\title{
Standards of Clinical Practice for Renal Pharmacists
}

\author{
Colette B Raymond, Lori D Wazny, and Amy R Sood
}

\section{INTRODUCTION}

$\mathrm{T}$ he prevalence of chronic kidney disease $(\mathrm{CKD})$ continues to increase. ${ }^{1}$ Patients with stage 1 to $5 \mathrm{CKD}$ and those undergoing dialysis are at extremely high risk for drug therapy problems (DTPs). ${ }^{2,3}$ In controlled trials involving general patient populations, clinical pharmacist interventions have reduced hospital admissions, length of hospital stay, readmissions, and emergency department visits. ${ }^{4-7}$ The activities of pharmacists most strongly associated with improved patient outcomes include participating on rounds, interviewing patients, performing medication reconciliation, counselling patients on discharge, and conducting postdischarge followup. ${ }^{5}$ A systematic review of 8 controlled trials involving patients with $\mathrm{CKD}$ showed that clinical pharmacist interventions improved management of anemia, blood pressure, and lipids, as well as calcium and phosphate parameters. ${ }^{8}$ In this patient population, clinical pharmacists' interventions reduced hospital admissions, length of hospital stay, and incidence of end-stage renal disease or death. ${ }^{8}$

The Manitoba Renal Program (MRP) provides comprehensive renal care throughout the province of Manitoba, Canada (population 1.2 million). The program provides care at 4 urban hospitals and 12 rural hemodialysis units. Health services offered include in-centre and home hemodialysis, peritoneal dialysis, and interprofessional renal health clinics for individuals with stage 1 to $5 \mathrm{CKD}$ who do not require renal replacement therapy. At the time this article was prepared, in mid-2013, the MRP had approximately 1100 hemodialysis patients, 285 peritoneal dialysis patients, and nearly 4500 patients with stage 1 to $5 \mathrm{CKD}$.

\section{DESCRIPTION OF PHARMACY PRACTICE MODEL}

The MRP pharmacists operate within a patient-centred medication therapy management model to provide care for patients with stage 1 to $5 \mathrm{CKD}$ and patients undergoing dialysis within the program. ${ }^{9}$ The MRP has a unique funding structure, with one full-time equivalent (FTE) clinical pharmacist for every 100 hemodialysis patients, 200 peritoneal dialysis or home hemodialysis patients, or 300 patients with stage 1 to 5 CKD. ${ }^{10}$ This funding structure provides equitable and consistent patient care across the province and allows the pharmacists to perform patient care, conduct research, and serve as educators. As of 2013, the MRP employed 19 individual pharmacists, whose time devoted to the program ranged from 0.2 to $1.0 \mathrm{FTE}$, for an overall total of $11.8 \mathrm{FTE}$ clinical pharmacists. On average, these pharmacists spend $90 \%$ (range $20 \%-100 \%$ ) of their MRP time performing activities related to direct patient care within the program, with the remainder of their time spent performing drug distribution in the hospital inpatient pharmacy. The MRP pharmacists attend all nephrologist clinics. In clinics for patients with stage 1 to $5 \mathrm{CKD}$, the pharmacists focus on those patients who have stage 4 or 5 $\mathrm{CKD}$, as well as patients with stage 1 to $3 \mathrm{CKD}$ who are receiving pharmacotherapy for glomerulonephritis. In clinics for peritoneal dialysis, home hemodialysis, and rural hemodialysis, the pharmacists see all patients. The pharmacists also staff the in-centre hemodialysis units at each urban hospital and liaise by telephone with the 16 rural hemodialysis units. The MRP pharmacists have a highly diverse practice, working at a variety of institutions that are geographically separate and that have different pharmacy managers, practice patterns, clinic structures, and patient populations; they also interact with different nephrologists within the MRP. However, to ensure consistency in patient care, the MRP pharmacists meet at least every 2 months in person and by teleconference to discuss the clinical and operational issues affecting them. Two of the pharmacists have postbaccalaureate Doctor of Pharmacy training, and they serve as clinical practice leaders for the other MRP pharmacists, focusing on hemodialysis and peritoneal dialysis, respectively. 


\section{DEVELOPMENT AND EVALUATION OF STANDARDS OF PRACTICE FOR THE MRP PHARMACISTS}

Working collaboratively with pharmacy managers, MRP pharmacists, and the MRP itself, we sought to develop standards of clinical practice for the MRP pharmacists. The purpose of doing so was to define and prioritize the core activities that these renal pharmacists must perform on a regular weekday with full staffing levels. We evaluated the literature describing the role of renal clinical pharmacists, surveyed MRP pharmacists about existing clinical pharmacist services, met with pharmacy and MRP stakeholders, and evaluated existing pharmacist standards of practice and existing activities and practices of the MRP pharmacists. ${ }^{11}$ A small working group of MRP pharmacists developed a draft set of

\section{Box 1. Standards of Clinical Practice for Renal Pharmacists in the Manitoba Renal Program (MRP)}

The pharmacist must perform these core clinical activities on fully staffed weekdays* (in order of priority):

1. Attend all MRP clinics (includes PD, home and rural HD, and CKD stages 1-5 clinics; total of 24 half-day clinics per week):

o Review laboratory test results and medications for all patients.

o Document in health record any recommendations, suggestions, or further patient information required for patients not seen by a pharmacist.

- For patients seen by a pharmacist, generate best possible medication history and perform medication reconciliation ${ }^{12}$ and detailed medication review.

2. Attend multidisciplinary patient care rounds (twice weekly for HD and PD patients):

o Contribute to interprofessional discussion about patients.

- Identify admitted patients for discharge medication reconciliation.

o Identify patients for medication review by a pharmacist.

3. Perform discharge (and transfer) medication reconciliation for admitted patients receiving dialysis before discharge or at first subsequent dialysis session (HD patients only). ${ }^{12}$

o Reconcile inpatient medications with home and in-centre HD medications.

o Perform detailed medication review (see Box 2) and document recommendations in the patient's medical record.

- Write discharge prescription for medications, including appropriate medications for in-centre HD and new medications started in hospital. Contact prescribing nephrologist to make recommendations and confirm prescription.

o Provide patient with medication card and counselling.

4. Review monthly laboratory test results for HD patients.

5. Perform detailed medication review for new starts to HD or PD (see Box 2) within 2 weeks.

6. Perform detailed medication review for other patients (see Box 2).

The pharmacist will perform the following "must do" activities (prioritized according to pharmacist's professional judgment):

- Ensure follow-up laboratory tests are ordered, according to pharmacist's recommendations.

- Ensure patients have adequate prescriptions and refills.

- Liaise with community pharmacy as appropriate (e.g., to facilitate prescription delivery, compliance aid, drug coverage).

- Liaise with patient, caregivers, family members, and other health care professionals as appropriate to provide medication-related information to or for patients.

- Provide drug information for immediate patient care that day.

- Provide education to pharmacy students and residents.

- Provide monitoring and follow-up for recommendations.t

- Provide communication between MRP and other pharmacists within the facility. $\dagger$

The pharmacist shall perform the following desirable activities as appropriate and as pharmacist is available:

- Participate in MRP and pharmacy program initiatives (e.g., development of drug protocols, review of preprinted orders, participation on committees, development of policy and procedures, responses to drug shortages).

- Provide education-related activities to health care professionals.

- Provide communication between MRP and other pharmacists at other facilities.

- Provide drug information not needed immediately.

- Perform drug-use management activities, including prospective audits.

- Participate in projects or research.

- Investigate medication incidents or errors.

- Review or triage medication orders to identify drug therapy problems related to appropriateness, duration, and dosing of each medication, as well as drug interactions (as an activity separate from medication review, medication reconciliation, or MRP clinic visit).

$\mathrm{CKD}=$ chronic kidney disease, $\mathrm{HD}=$ hemodialysis, $\mathrm{PD}=$ peritoneal dialysis

*Evening HD patients are reviewed and seen by pharmacists who work later shifts periodically, in order that all patients are seen by a pharmacist. Weekend pharmacist coverage consists of centralized dispensary pharmacist coverage at each site.

tWhen the pharmacy is short-staffed, these are considered "should do" (rather than "must do") activities. 
Box 2. Steps in Review of Patients with Chronic Kidney Disease for Drug Therapy Problems (DTPs)

General medication review:

- For new dialysis patients, before nephrologist review or clinic visit (every 6 months to 1 year) or at the request of another health care professional (see Appendix 1)

- Interview patient, caregivers, family members, and other health care professionals

- Generate best possible medication history and perform medication reconciliation ${ }^{12}$

- Review laboratory test results, investigations, physical findings, and medications to identify DTPs

- Document medication review, DTPs, and recommendations in the medical record

- Identify and resolve actual/potential DTPs during discharges, medication reviews, clinic visits, between clinic visits (after review of laboratory test results), on medication order review, or detailed medication review

Assess patient for general DTPs ${ }^{13-19}$.

- allergies and intolerances

- drug-drug interactions

- adverse drug reactions

- medication causing or exacerbating a symptom

- duplication of pharmacologically or therapeutically similar medications

- appropriate dosage form and route of administration

- medication therapy not indicated

- medication indicated but not utilized

medication adherence

problems related to IV administration

- medications that require renal dose adjustments

- medications that are contraindicated in CKD or that should be minimized

medications that are no longer required in dialysis

Assess patient for DTPs specific to CKD by assessing the following:

- Anemia: Assess hemoglobin, transferrin saturation, ferritin, use of erythropoietic-stimulating agent, iron, and renal multivitamin. Consider erythropoietin hyporesponsiveness. ${ }^{20-25}$

- Mineral and bone disease: Assess corrected calcium, serum phosphate, parathyroid hormone, alkaline phosphatase, albumin, calcium bath concentration, phosphate and calcium additives to the dialysate, surgical history (for parathyroidectomy), use of phosphate binders, vitamin D analogue, or cinacalcet. Liaise with dietitian about diet. ${ }^{26,27}$

- Cardiovascular risk: Assess for presence of cardiovascular disease and risk factors, and therapies to reduce this risk (antiplatelets, anticoagulants, antihypertensives, statins, antianginal therapies, and antiarrhythmics). ${ }^{27-29}$

- Hypertension and proteinuria: Assess blood pressure before, during, and after dialysis, in clinic and at home; assess dry weight, proteinuria, antihypertensives, and antiproteinuric therapies. ${ }^{27,30}$

- Diabetes mellitus: Assess glucose monitoring before and after dialysis, in clinic and at home, as well as glycated hemoglobin and use of hypoglycemic agents. ${ }^{27,31-33}$

- Pain: Assess source of pain, its quantity and quality, and use of opioids, NSAIDs, and adjunctive therapies. ${ }^{34,35}$

- Peripheral neuropathy: Assess source of pain, its quantity and quality, and use of antidepressants, anticonvulsants, and opioids. ${ }^{36}$

- Restless leg syndrome: Assess symptom severity and frequency, sleep disturbance, daytime fatigue, and use of dopamine agonists, gabapentin, levodopa, benzodiazepines, and opioids. ${ }^{37,38}$

- Smoking status: Assess readiness to quit and use of nicotine replacement therapy, bupropion, or varenicline; provide education. ${ }^{39}$

- Cramps: Assess symptom severity and frequency, as well as use of quinine or vitamin $\mathrm{E}^{40}$

- Pruritus: Assess symptom severity and use of topical or systemic agents.

- Gastrointestinal issues (e.g., reflux, history of bleeding, ulcer, dyspepsia, constipation, diarrhea): Assess signs and symptoms, as well as use of antacids, laxatives, stool softeners, agents to treat diarrhea, NSAIDs, or corticosteroids.

- Infectious diseases (e.g., IV catheter-related infections, skin infections, peritonitis) requiring treatment or prophylaxis, including antibiotic locks and intraperitoneal antibiotics: Assess signs and symptoms, as well as culture and sensitivity results. ${ }^{41-43}$

- Hyperkalemia (for stage 1-5 CKD patients): Assess serum potassium, presence of hemolysed sample, and use of potassium supplements, ACE inhibitors, ARBs, potassium-sparing diuretics, and other agents known to increase serum potassium. Assess use of potassium-binding resins and diuretics. Liaise with dietitian regarding diet. ${ }^{44}$

- Metabolic acidosis (for stage 1-5 CKD patients): Assess serum bicarbonate concentrations and use of supplementation. ${ }^{27}$

- Depression, anxiety, insomnia: Assess consultations with other health care professionals and use of antidepressants, antipsychotics, benzodiazepines, and sedatives. ${ }^{45}$

- Gout (for patients with stage 1-5 CKD): Assess serum uric acid level, frequency and severity of gout attacks, and use of colchicine, NSAIDs or corticosteroids, allopurinol, and febuxostat. ${ }^{46}$

- Review patient for the use of the following high-alert medications: digoxin, lithium, phenytoin, immunosuppressive therapy.

- Assess serology and vaccination status for hepatitis B, pneumonia, and influenza. ${ }^{27,47}$

$\mathrm{ACE}=$ angiotensin-converting enzyme, $\mathrm{ARB}=$ angiotensin receptor blockers, $\mathrm{CKD}=$ chronic kidney disease, NSAID = nonsteroidal anti-inflammatory drug. 
standards of clinical practice for renal pharmacists. The draft was distributed to all MRP pharmacists on multiple occasions to obtain feedback. Feedback for priority activities was also obtained from nephrologists. Consensus was achieved, and all MRP pharmacists, pharmacy managers, and nephrologist medical directors have adopted the final version of the standards of clinical practice for renal pharmacists (Box 1). These standards specify that MRP pharmacists should routinely evaluate their patients for the DTPs commonly experienced by people with CKD (listed in Box 2). The standards of clinical practice can be updated to reflect the incorporation of local policies and procedures, patient safety initiatives, and published guidelines.

\section{IMPLICATIONS FOR PRACTICE}

Creation of standards of clinical practice for renal pharmacists across diverse practice environments and numerous pharmacists has allowed for a common method to perform and prioritize clinical pharmacist activities and to aid in the training of new staff. Across the MRP, the pharmacists typically assess patients before the nephrologist does so. Therefore, the pharmacist's documentation is critical to ensuring that an accurate medication list is included in the chart and that DTPs are identified before the nephrologist's review. This streamlined approach helps to resolve existing DTPs quickly and prevents additional DTPs from occurring. The use of standards of practice as a common approach to patient assessment provides continuity of pharmacist care across the MRP. For example, the standards of practice have been used to develop a standard template for medication review for patients undergoing hemodialysis or peritoneal dialysis, which becomes part of the medical record (see Appendix 1). Within the MRP, we have used the standards of practice as guidelines and for training purposes. The standards could also be used to develop criteria for competency assessment or to inform performance appraisals.

Others have developed and validated a list of criteria to assess medication safety and use issues in patients with CKD in order to identify DTPs. ${ }^{48}$ However, that list of DTPs was based on interventions by community pharmacists. The specialized renal pharmacists have the advantage of access to patient care records and have developed trusting relationships with the nephrologists, both of which facilitate optimization of medication therapy. The renal pharmacist standards of practice document describes renal-specific DTPs, as well as processes and priorities for renal pharmacists functioning as members of an interprofessional team.

\section{CONCLUSIONS}

The standards of practice for renal pharmacists developed within the MRP are a unique set of evidence-based practice guidelines that can serve to educate and train renal pharmacists, students, or trainees completing a renal pharmacy rotation. Furthermore, the standards of practice can serve as a tool to standardize patient care, set priorities, develop criteria for competency assessment, and inform performance appraisals for renal pharmacists. Additionally, centres without renal pharmacists on staff could use the standards of practice to justify the funding needed to hire such specialized practitioners.

\section{References}

1. Coresh J, Selvin E, Stevens LA, Manzi J, Jusek JW, Eggers P, et al. Prevalence of chronic kidney disease in the United States. JAMA. 2007;298(17):2038-47.

2. Manley HJ, Cannella CA, Bailie GR, St Peter WL. Medication-related problems in ambulatory hemodialysis patients: a pooled analysis. Am J Kidney Dis. 2005;46(4):669-80.

3. Zillich AJ, Saseen JJ, Dehart RM, Dumo P, Grabe DW, Gilmartin C, et al. Caring for patients with chronic kidney disease: a joint opinion of the ambulatory care and the nephrology practice and research networks of the American College of Clinical Pharmacy. Pharmacotherapy. 2005; 25(1):123-43.

4. Chisholm-Burns MA, Kim Lee J, Spivey CA, Slack M, Herrier RN, HallLipsy E, et al. US pharmacists' effect as team members on patient care: systematic review and meta-analyses. Med Care. 2010;48(10):923-33.

5. Kaboli PJ, Hoth AB, McClimon BJ, Schnipper JL. Clinical pharmacists and inpatient medical care. Arch Intern Med. 2006;166(9):955-64.

6. Makowsky MJ, Koshman SL, Midodzi WK, Tsuyuki RT. Capturing outcomes of clinical activities performed by a rounding pharmacist participating in a team environment. The COLLABORATE study. Med Care. 2009; 47(6):642-50.

7. Gillespie U, Alassaad A, Henrohn D, Garmo H, Hammarlund-Udenaes $\mathrm{M}$, Toss $\mathrm{H}$, et al. A comprehensive pharmacist intervention to reduce morbidity in patients 80 years or older: a randomized controlled trial. Arch Intern Med. 2009;169(9):894-900.

8. Salgado TM, Moles R, Benrimoj SI, Fernandez-Llimos F. Pharmacists' interventions in the management of patients with chronic kidney disease: a systematic review. Nephrol Dial Transplant. 2012;27(1):276-92.

9. Medication therapy management in pharmacy practice. Core elements of an MTM service model. Version 2.0. Washington (DC): American Pharmacists Association and National Association of Chain Drug Stores Foundation; 2008 [cited 2013 Mar 26]. Available from: www.pharmacist.com/ sites/default/files/files/core_elements_of_an_mtm_practice.pdf

10. Raymond CB, Wazny LD, Sood A, Vercaigne L. Establishing and funding renal clinical pharmacy services. Nephrol News Issues. 2010; 24(6):40-1,45-7.

11. Direct patient care guidelines: pharmacist performance expectations for acute care facilities. Winnipeg (MB): Winnipeg Regional Health Authority Pharmacy Program; 2004.

12. Manitoba Renal Program procedure [60.40.09]: Guidelines for medication reconciliation. Winnipeg (MB): Winnipeg Regional Health Authority; 2011 Nov [cited 2013 Mar 27]. Available from: www.kidneyhealth.ca/ wp/wp-content/uploads/pdfs/P\&P/P\&P_60.40.09_guideline.pdf

13. Strand LM, Morley PC, Cipolle RJ, Ramsey R, Lamsam GD. Drug-related problems: their structure and function. DICP. 1990;24(11):1093-7.

14. Cipolle RJ, Strand LM, Morley PC. Pharmaceutical care practice: the clinician's guide. 2nd ed. New York (NY): McGraw-Hill; 2004.

15. Bailie GR, Mason NA. 2012 dialysis of drugs. Saline (MI): Renal Pharmacy Consultants, LLC; 2012.

16. Aronoff GR, Berns JS, Brier ME, Golper TA, Morrison G, Singer I, et al. Drug prescribing in renal failure guidelines for adults. 5th ed. Portland (OR): Book News, Inc; 2007.

17. Regional policy [110.160.010]: Assessment of initial medication orders for appropriate dosing based on renal function. Winnipeg (MB): Winnipeg Regional Health Authority; 2007 Oct.

18. Matzke GR, Aronoff GR, Atkinson AJ, Bennett WM, Decker BS, Echardt KU. Drug dosing consideration in patients with acute and chronic kidney disease- a clinical update from Kidney Disease: Improving Global Outcomes (KDIGO). Kidney Int. 2011;80(11):1122-37. 
19. Raymond CB, Wazny LD, Sood AR. Medication adherence in patients with chronic kidney disease. CANNT J. 2011;21(2):47-50.

20. Moist LM, Foley RN, Barrett BJ, Madore F, White CT, Klarenbach SW, et al. Clinical practice guidelines for evidence-based use of erythropoieticstimulating agents. Kidney Int. 2008;74 Suppl 110:S12-8.

21. National Kidney Foundation. KDOQI clinical practice guidelines and clinical practice recommendations for anemia in chronic kidney disease. Am J Kidney Dis. 2006;47 Suppl 3:S1-146.

22. KDOQI. KDOQI clinical practice guideline and clinical practice recommendations for anemia in chronic kidney disease: 2007 update of hemoglobin target. Am J Kidney Dis. 2007;50(3):471-530.

23. Kidney Disease: Improving Global Outcomes (KDIGO) Anemia Work Group. KDIGO clinical practice guideline for anemia in chronic kidney disease. Kidney Int Suppl. 2012;2(4):279-335.

24. Levin A, Hemmelgarn B, Culleton B, Tobe S, McFarlane P, Ruzica M, et al. Guidelines for the management of chronic kidney disease CMAJ. 2008;179(11):1154-62.

25. Bennett CL, Becker PS, Kraut EH, Samaras AT, West DP. Intersecting guidelines: administering erythropoiesis-stimulating agents to chronic kidney disease patients with cancer. Semin Dial. 2009;22(1):1-4.

26. Kidney Disease: Improving Global Outcomes (KDIGO) CKD-MBD Work Group. KDIGO clinical practice guideline for the diagnosis, evaluation, prevention, and treatment of chronic kidney disease - mineral and bone disorder (CKD-MBD). Kidney Int. 2009;76 Suppl 113:S1-S130.

27. Kidney Disease: Improving Global Outcomes (KDIGO) CKD Work Group. KDIGO 2012 clinical practice guideline for the evaluation and management of chronic kidney disease. Kidney Int Suppl. 2013;3(1):1-150.

28. Herzog CA, Asinger RW, Berger AK, Charytan DM, Díez J, Hart RG, et al. Cardiovascular disease in chronic kidney disease. A clinical update from Kidney Disease: Improving Global Outcomes (KDIGO). Kidney Int. 2011;80(6):572-86.

29. Bell AD, Roussin A, Cartier R, Chan WS, Douketis JD, Gupta A. The use of antiplatelet therapy in the outpatient setting: Canadian Cardiovascular Society Guidelines executive summary. Can J Cardiol. 2011;27(2):208-21.

30. Kidney Disease: Improving Global Outcomes (KDIGO) Blood Pressure Work Group. KDIGO clinical practice guideline for the management of blood pressure in chronic kidney disease. Kidney Int Suppl. 2012; 2(5):337-414.

31. Canadian Diabetes Association Clinical Practice Guidelines Expert Committee. Canadian Diabetes Association 2013 clinical practice guidelines for the prevention and management of diabetes in Canada. Can J Diabetes. 2013;37 Suppl 1:S1-212.

32. National Kidney Foundation. KDOQI clinical practice guidelines and clinical practice recommendations for diabetes and chronic kidney disease. Am J Kidney Dis. 2007:49 Suppl 2:S1-180.

33. National Kidney Foundation. KDOQI clinical practice guideline for diabetes and CKD: 2012 update. Am J Kidney Dis. 2012;60(5):850-86.

34. Davison SN. The prevalence and management of chronic pain in end-stage renal disease. J Palliat Med. 2007;10(6):1277-87.

35. Canadian guideline for safe and effective use of opioids for chronic non-cancer pain. Hamilton (ON): National Opioid Use Guideline Group; 2010 [cited 2013 Mar 26]. Available from: http://national paincentre.mcmaster.ca/opioid/

36. Naylor HK, Raymond CB. Treatment of neuropathic pain in patients with chronic kidney disease. CANNT J. 2011;21(1):34-40.

37. Raymond CB, Breland L, Wazny LD, Sood AR, Orsulak CD. Treatment of restless legs syndrome in patients receiving dialysis-a focus on medications. CANNT J. 2010;20(2):29-35.

38. Walters AS, LeBrocq C, Dhar A, Hening W, Rosen R, Allen RP, et al.; International Restless Legs Syndrome Study Group. Validation of the International Restless Legs Syndrome Study Group rating scale for restless legs syndrome. Sleep Med. 2003;4(2):121-32.

39. Raymond CB, Naylor H. Strategies for smoking cessation in patients with chronic kidney disease. CANNT J. 2010;20(4):24-31.

40. Raymond CB, Wazny LD. Treatment of leg cramps in patients with chronic kidney disease receiving hemodialysis. CANNT J. 2011;21(3):19-21.
41. Zelenitsky SA, Ariano RE, McCrae ML, Vercaigne LM. Initial vancomycin dosing protocol to achieve therapeutic serum concentrations in patients undergoing hemodialysis. Clin Infect Dis. 2012;55(4):527-33.

42. de Vin F, Rutherford P, Faict D. Intraperitoneal administration of drugs in peritoneal dialysis patients: a review of compatibility and guidance for clinical use. Perit Dial Int. 2009;29(1):5-15.

43. Li PK, Szeto CC, Piraino B, Bernardini J, Figueiredo AE, Gupta A, et al. Peritoneal dialysis-related infections recommendations: 2010 update. Perit Dial Int. 2010;30(4):393-423.

44. Raymond CB, Sood AR, Wazny LD. Treatment of hyperkalemia in patients with chronic kidney disease- $\mathrm{a}$ focus on medications. CANNT J. 2010;20(3):49-54.

45. Hedayati SS, Yalamanchili V, Finkelstein FO. A practical approach to the treatment of depression in patients with chronic kidney disease and end-stage renal disease. Kidney Int. 2012;81(3):247-55.

46. Abdellatif AA, Elkhalili N. Management of gouty arthritis in patients with chronic kidney disease. Am J Ther. 2012 Sep 6. [Epub ahead of print].

47. Manitoba Renal Program protocol [60.30.03]: Adult patient screening and vaccination protocol for hepatitis $B$ and hepatitis C. Winnipeg (MB): Winnipeg Regional Health Authority; 2011 Nov [cited 2013 Mar 27]. Available from: www.kidneyhealth.ca/wp/wp-content/uploads/pdfs/P\&P/ P\&P_60.30.04.pdf

48. Desrochers JF, Lemieux JP, Morin-Belanger C, Paradis FS, Berbiche D, Barcena PQ, et al. Development and validation of the PAIR (pharmacotherapy assessment in chronic renal disease) criteria to assess medication safety and use issues in patients with CKD. Am J Kidney Dis. 2011;58(4):527-35.

Colette B Raymond, PharmD, MSc, ACPR, was, at the time of writing, with the Winnipeg Regional Health Authority Pharmacy Program, Department of Pharmaceutical Services, Health Sciences Centre, Winnipeg, Manitoba. She is now with the Department of Pharmacy, University Health Network, and the Leslie Dan Faculty of Pharmacy, University of Toronto, Toronto, Ontario.

Lori D Wazny, PharmD, is with the Winnipeg Regional Health Authority Pharmacy Program, Department of Pharmaceutical Services, Health Sciences Centre, Winnipeg, Manitoba.

Amy R Sood, BScPhm, PharmD, is with the Manitoba Renal Program, Department of Pharmacy, St Boniface Hospital, Winnipeg, Manitoba.

Competing interests: Colette Raymond is a member of the Canadian Hospital Pharmacy Residency Board and has received grants for work unrelated to this article from the Canadian Institutes of Health Research and Health Canada. No competing interests declared by Lori Wazny or Amy Sood.

\section{Address correspondence to:}

Dr Lori D Wazny

Winnipeg Regional Health Authority Pharmacy Program

Department of Pharmaceutical Services

Health Sciences Centre

820 Sherbrook Street, Room MS-189

Winnipeg MB R3A 1R9

e-mail: Iwazny@hsc.mb.ca

\section{Acknowledgements}

The efforts of all of the Manitoba Renal Program pharmacists who contributed to developing the standards of clinical practice are greatly appreciated. The contributions of Nick Honcharik, who spearheaded development of the original Winnipeg Regional Health Authority's clinical practice expectations for pharmacists, are acknowledged. This work was funded by the Winnipeg Regional Health Authority and the Manitoba Renal Program.

See page 374 for Appendix 1 
Appendix 1. Template for medication review performed by pharmacists in the Manitoba Renal Program. Copyright (C) 2013 Manitoba Renal Program. Reproduced by permission. ACE/ARB = angiotensin-converting enzyme inhibitor/angiotensin II receptor blocker, A.Fib = atrial fibrillation, ALKPhos = alkaline phosphatase, $\mathrm{ASA}=$ acetylsalicylic acid, $\mathrm{BB}=$ beta-blocker, $\mathrm{BP}=$ blood pressure, $\mathrm{B} . \mathrm{S} .=$ blood sugar, $\mathrm{Ca}{ }^{2}++=\mathrm{calcium}, \mathrm{CCB}=$ calcium channel blocker, $\mathrm{CHF}=$ congestive heart failure, $\mathrm{CorCa}=$ calcium corrected for albumin, $\mathrm{CVA}=$ cerebrovascular accident, $\mathrm{ESA}=$ erythropoiesis-stimulating agent, ESRD = end-stage renal disease, GGT = gamma-glutamyl transferase, HbA1C = glycosylated hemoglobin, HD = hemodialysis, $\mathrm{HDL}=$ high-density lipoprotein, $\mathrm{Hgb}=$ hemoglobin, $\mathrm{HR}=$ heart rate, $\mathrm{HTN}=$ hypertension, INR = international normalized ratio, IV = intravenous, $\mathrm{LDL}=$ low-density lipoprotein, $\mathrm{Ml}=$ myocardial infarction, NTG = nitroglycerin, OHA = oral hypoglycemic agent, OTC = over-the-counter, $\mathrm{PO} 4=$ phosphate, $\mathrm{PTH}=$ parathyroid hormone, $\mathrm{RLS}=$ restless leg syndrome, $\mathrm{Rx}=$ prescription, $\mathrm{TC}=$ total cholesterol, $\mathrm{TG}=$ triglycerides, $\mathrm{TIA}=$ transient ischemic attack, TSAT = transferrin saturation .

Date:

Seen: In Unit Clinic Site Visit

Compliance Tools: Bubble Pack Dosette Other:

Community Pharmacy: Potassium binding resin at home Yes No

Medications verified with: Patient/Caregiver Rx Label Pharmacy

Electronic prescription record Chart reviewed

Herbal Products: No Yes

OTC (other than as Rx): No Yes

Allergies/Intolerances:

ESRD Secondary to:

HD initiated on:

Comments:

\section{Anemia:}

$\mathrm{Hgb}$

TSAT

Ferritin

Mineral Metabolism:

CorCa

$\mathrm{PO} 4$

ALKPhos/GGT

$\mathrm{Ca}^{2}++$ bath
ESA

IV iron

Replavite Yes No

PO4 binders

PTH

Vitamin D

Parathyroidectomy No Yes

\section{Cardiovascular disease:}

History of: HTN Diabetes CVATIA MI A.fib CHF

Smoking Angina

Pre HD BP: Post HD BP: HR

Lipid profile: (date)TC $\mathrm{HDL}$ LDL TG TC/HDL

BB ACE/ARB ASA Clopidogrel

Statin $C C B$ Warfarin Diuretic (INR target

NTG Spray

\section{Diabetes:}

12 HbA1c: __ (date) Ophthalmologist Endocrinologist

No Pre/Post HD glucose: B.S.<4: No Yes Insulin $\mathrm{OHA}$ :

\section{Gastrointestinal Issues:}

\section{RLS/Leg Cramps:}

\section{Pruritus}

Sleep Disturbances:

Pain Issues:

Therapeutic Drug Monitoring:

Other Issues:

Pharmacist Signature: Date: 